\title{
An insertion/deletion polymorphism at the microRNA-122 binding site in the interleukin-1 $\alpha 3$ '-untranslated region is associated with a risk for osteoarthritis
}

\author{
FAN YANG ${ }^{1,2}$, ANFENG HU $^{3}$, DEWEI ZHAO ${ }^{1}$, LIN GUO $^{1}$, LEI YANG $^{1}$, \\ BENJIE WANG $^{1}$, FENGDE TIAN ${ }^{1}$, BAOYI LIU ${ }^{1}$, SHIBO HUANG ${ }^{1}$ and HUI XIE ${ }^{1}$ \\ ${ }^{1}$ Department of Orthopedics, Zhongshan Hospital of Dalian University, Dalian, Liaoning 116001; \\ ${ }^{2}$ Department of Orthopedics, Graduate School of Zunyi Medical University, Zunyi, Guizhou 563000; \\ ${ }^{3}$ Department of Orthopedics, The People's Hospital of Zhangqiu, Zhangqiu, Shandong 250200, P.R. China
}

Received September 1, 2014; Accepted May 13, 2015

DOI: $10.3892 / \mathrm{mmr} .2015 .4121$

\begin{abstract}
Polymorphisms located at microRNA (miRNA) binding sites may affect the expression of genes. The present study aimed to identify the association between an insertion/deletion (Ins/Del) polymorphism (rs3783553) in the 3'-untranslated region (3'-UTR) of interleukin-1 $\alpha$ (IL-1A) and the risk for osteoarthritis (OA). Using a luciferase reporter system, IL-1A was identified in the present study as an effective target gene of miR-122 in synovial cells that were obtained from patients who had received a synovectomy. This finding was verified further by the observation that exogenous over-expression of miR-122 in the synovial cells significantly downregulated the expression of IL-1A in the cells with Ins/Ins and Ins/Del genotypes, but not in the cells with Del/Del genotypes. Patients with OA $(n=931)$ and OA-free volunteers $(n=952)$ were enrolled in the study. Compared with the Del/Del genotype, patients possessing the Ins/Del or Ins/Ins genotype were associated with a lower risk for $\mathrm{OA}$ [odds ratio $(\mathrm{OR})=0.67, \mathrm{P}=0.0051 ; \mathrm{OR}=0.65$, $\mathrm{P}=0.0031$, respectively], and the association was even stronger in young subjects $(<62$ years $)(\mathrm{OR}=0.53 ; \mathrm{P}<0.001)$. Additionally, it was found that genotype was associated with radiographic severity $(\mathrm{OR}=0.72 ; \mathrm{P}=0.023)$. The synovial fluid $(\mathrm{SF})$ concentrations of IL-1A and miR-122 were measured in 75 OA patients. While the miR-122 concentrations were found to be comparable between each genotype group, the SF concentration of IL-1A in the Del/Del group was significantly higher than in the Ins/Del and Ins/Ins genotype groups. Therefore, the present study identified that the Ins/Del polymorphism in the 3'-UTR of IL-1A may affect genetic expression and, to the best of our knowledge,
\end{abstract}

Correspondence to: Dr Dewei Zhao, Department of Orthopedics, Zhongshan Hospital of Dalian University, 6 Jiefang Street, Dalian, Liaoning 116001, P.R. China

E-mail: dewei_zhao@163.com

Key words: miR-122, insertion, deletion, osteoarthritis, interleukin-1 $\alpha$ is the first study to demonstrate that the minor allele (Del) is associated with an elevated risk for OA and disease severity.

\section{Introduction}

Osteoarthritis (OA) is a disabling and progressive disease that affects multiple joints. Patients with OA constitute a substantial medical, social and economic burden, therefore it is necessary to conduct investigations to clarify the etiological and predictive factors. There are two predominant types of OA; primary OA, which is characterized by its late onset, and secondary $\mathrm{OA}$, which is differentiated from primary OA by its early onset with established reasons, such as developmental abnormalities and trauma. However, the causes for developing primary OA have not yet been fully elucidated. It is hypothesized that the risk of primary OA involves a variety of factors, including gender, age, behavior, obesity and ethnicity, as well as genetic factors $(1,2)$. Results of linkage analysis and familial aggregation indicate that complex genetic inheritance is critical in the underlying pathogenesis of primary OA (3-6). The interleukin-1 (IL-1) gene cluster on chromosome 2 (nucleotide position, $113531492-113891593$; 2q13-2q14.1) may serve as one of the genetic loci that confer risk. IL-1 is a multifunctional cytokine that predominantly impacts on inflammation and immunity. In addition, IL-1 acts as a regulator of the homeostatic status of the human body and is critical in the development of diseases $(7,8)$. Indeed, IL-1 is a family of three associated proteins that are encoded by different genes. The family comprises two major agonistic cytokines, interleukin-1 $\alpha$ (IL-1A) and interleukin- $1 \beta$ (IL-1B), as well as their endogenous receptor antagonist, interleukin-1 receptor (IL-1R). It has been shown that the potency of IL-1A, instead of IL-1B, in synovial fluid (SF) was positively correlated with the occurrence of OA and that joint tissues exposed to IL-1A in vitro were significantly damaged, which triggered the development of $\mathrm{OA}$ in a rabbit arthritis model $(9,10)$. Evidence from numerous studies has indicated that the polymorphisms or haplotypes in the IL-1A gene were significantly associated with a risk for OA (11-13).

MicroRNAs (miRNAs) represent an abundant class of short non-coding RNAs that act as post-transcriptional regulators. 
To date, experiments have identified 600 human miRNAs, modulating more than one-third of cellular messenger RNAs (mRNAs) (14). Increasing evidence has revealed that dysregulated expression patterns of miRNAs were associated with various diseases, including cancer, neurodegenerative and cardiovascular diseases, and viral infections $(14,15)$. Binding of miRNA to mRNA is important in the regulation of mRNA stability, as well as translation efficiency. However, this binding may be compromised by the polymorphisms that are located within miRNA target sites, which may either abrogate existent binding sites or generate illegitimate binding sites (16-18), and impact on the development of many diseases, such as various types of cancer (19-21), Parkinson's disease (22), and other disorders (23-25). A previous study by Gao et al revealed that the binding of miR-122 and the regulation of IL-1A expression by the miRNA were significantly affected by a 2-thiothiazolidine-4-carboxylic acid (TTCA) insertion/deletion (Ins/Del) polymorphism (rs3783553) that was residing in the IL-1A 3'-untranslated region (UTR), which conferred a significantly increased risk for hepatocellular carcinoma in a Chinese population (26).

Thus, the aim of the present study was to demonstrate the functional influence of the rs3783553 polymorphism on the interaction between miR-122 and IL-1A 3'-UTR. Furthermore, the investigation of a Chinese Han population was conducted to evaluate the association between the rs3783553 polymorphism and the risk for OA, as well as the level of IL-1A in the SF of OA patients.

\section{Materials and methods}

Subjects. A total of 931 Chinese Han OA patients with clinically and radiologically confirmed diagnoses of OA were recruited to the Department of Orthopedics, at Zhongshan Hospital of Dalian University (Dalian, China). There were 317 females (34\%) and 614 males (66\%; average age, $63.2 \pm 12.9$ years). Informed consent was obtained prior to the donation of blood for the purposes of research. The radiographic criteria for OA were as follows: A Kellgren-Lawrence (KL) grade $>1$ of at least one knee; and being aged $>38$ years. The clinical criteria followed the guidelines of the American College of Rheumatology $(27,28)$ in order to classify the type of OA, and the Western Ontario and McMaster Universities Osteoarthritis Index (29) was used to evaluate pain and joint stiffness, as well as physical function in the OA patients. Patients were excluded from the study as follows: If they had a history of corticosteroid use; if they had undergone a bilateral knee replacement; if they exhibited other types of arthritis, cancer or other chronic diseases beyond hypertension or hypercholesterolemia. The KL grade (0-4) was recorded after radiographs had been obtained; a KL score of 1 or 2 grouped the patient into 'mild OA' and a KL score of 3 or 4 grouped the patient into 'severe OA'. A total of 952 healthy individuals (37\% female; mean age, $62.6 \pm 11.2$ years) who exhibited no clinical manifestation of OA were assigned to the control group. Written consent was obtained from each participant, and the study was approved by the medical ethics committees of Dalian University.

Collection of tissues and cell culture. Sterile synovial tissues were obtained during therapeutic open joint surgery from patients who had undergone a synovectomy. The synovial tissue was obtained, digested and cultured according to a previously reported protocol (30). Briefly, within $2 \mathrm{~h}$ after surgery, the sample was washed three times, and the superficial layer of synovium $\left(\sim 2 \mathrm{~mm}^{3}\right)$ was cut and placed in Dulbecco's modified Eagle's medium (DMEM; Gibco BRL, Eggenstein, Germany), supplemented with penicillin and streptomycin (Gibco BRL) per milliliter, and containing $4 \mathrm{mg} / \mathrm{ml}$ of clostridiopeptidase A (Sigma-Aldrich, St. Louis, MO, USA). The tissue fragments were sliced further with scissors and incubated for $5 \mathrm{~h}$ in $25 \mathrm{ml}$ DMEM at $37^{\circ} \mathrm{C}$ in a humidified atmosphere of $5 \%$ carbon dioxide. Subsequently, an equal volume of $0.05 \%$ trypsin and $0.02 \%$ EDTA in modified Puck's Saline A (Gibco-BRL) was added and incubation continued for another $2 \mathrm{~h}$ under the same conditions. The digested cells were centrifuged for $10 \mathrm{~min}$ at $400 \mathrm{x} \mathrm{g}$ at room temperature and the pellet $(1-2 \mathrm{ml})$ was suspended in DMEM (20-40 ml) supplemented with $10 \%$ fetal bovine serum (Gibco-BRL). The cells were passaged after the treatment with trypsin, split 1:3 and plated in $6-\mathrm{cm}$ polystyrene petri dishes at $5 \times 10^{5}$ cells per dish, containing $2 \mathrm{ml}$ medium.

Extraction and genotyping of DNA. Genomic DNA was extracted using a DNA extraction kit (Sangong Biotech, Shanghai, China). A routine polymerase chain reaction (PCR) using a PTC-200 cycler (Bio-Rad Laboratories, Inc., Hercules, CA, USA) was performed to amplify the fragment containing the TTCA Ins/Del polymorphism, rs3783553, with the following primers: Forward, 5'-ATTGGTCCGATC TTTGACTC-3' and reverse, 5'-TGATAACAGTGGTCT CATGG-3'. The PCR products underwent genotyping using a direct sequencing DNA sequencer 3730 (Applied Biosystems Life Technologies, Foster City, CA, USA).

Measurement of IL-1A in SF. SF was obtained from 75 OA patients by either direct aspiration $(n=52)$ or lavage $(n=23)$ and the samples were analyzed blinded to the clinical information. The levels of cytokines ( $\mathrm{ng} / \mathrm{ml}$ ) were determined by multiplex bead assay (Bio-Rad Laboratories, Inc.). The SF samples were spun down to remove cell debris, and divided into equal quantities and stored at $80^{\circ} \mathrm{C}$. The concentrations of IL-1A in the SF samples were determined by commercially available ELISA kits (MyBiosource LLC, San Diego, CA, USA) and the assays were performed according to the manufacturer's instructions.

Reverse transcription (RT) and quantitation of miR-122 in $S F$ by real-time PCR ( $q P C R)$. The method was conducted as previously described (31). Briefly, mRNA was reverse transcribed into cDNA using a NCode VILO miRNA cDNA Synthesis kit (Invitrogen Life Technologies, Carlsbad, CA, USA), and qPCR was performed on an Applied Biosystems 7900HT Real-Time PCR system (Applied Biosystems Life Technologies) with standard plasmids. The preliminary qPCR products underwent TA cloning using a TA cloning kit (Invitrogen Life Technologies) and the sequences of the inserted nucleotides were verified. Plasmids with known copy numbers were subject to qPCR, and standard curves for each of the miRNAs were generated. Absolute copy numbers of the target miRNA in the SF samples were obtained according to the generated standard curves. 
qPCR analysis of mRNA levels. Total RNA was isolated from the synovial cells (obtained from patients of different genotypes) with or without being transfected with miR-122 mimics using an Ambion RNA isolation kit (Ambion Life Technologies). This was subsequently converted to complementary DNA using an oligo (dT) 15 primer and SuperScript II Reverse Transcriptase (Invitrogen Life Technologies). TaqMan ${ }^{\circledR}$ Gene Expression assays (Applied Biosystems Life Technologies) were conducted using an ABI 7900HT Real-Time PCR system (Applied Biosystems Life Technologies) to quantify the relative IL-1A and miR-122 expression in the samples. U6 (RNU6-6P RNA) served as the internal control. All reactions were run in triplicate to reduce confounding variance.

Western blot analysis. Equal quantities of cell lysates were loaded onto SDS-polyacrylamide gel for electrophoresis. The separated proteins were subsequently transferred onto a polyvinylidene difluoride (PVDF) membrane, followed by blocking at room temperature for $1 \mathrm{~h}$ with Tris-buffered saline and Tween-20 [10 mM Tris- $\mathrm{Cl}$ (pH 8.0), $150 \mathrm{mM} \mathrm{NaCl}$ and $0.05 \%$ Tween-20) containing 5\% nonfat dry milk powder. The PVDF membrane was incubated with the rabbit anti-IL-1A polyclonal antibody (cat. no. AV54322; Sigma-Aldrich; dilution, 1:2,000) at $4^{\circ} \mathrm{C}$ overnight, followed by incubation with horseradish peroxidase-conjugated anti-rabbit secondary antibody (cat. no. A0545; Sigma-Aldrich; dilution, 1:10,000) at room temperature for $2 \mathrm{~h}$. The chemical fluorescence signal was detected using an enhanced chemiluminescence kit (GE Healthcare Life Sciences, Chalfont, UK) according to the manufacturer's instructions. A band of $\beta$-actin served to normalize the result of the target gene.

IL-IA 3'-UTR luciferase assay. The full length of human IL-1A 3'-UTR containing the gene locus (rs3783553) was PCR amplified using the following primer sequence obtained from a genomic DNA sample that was isolated from a wild-type subject: Forward, 5'-GATCTCTAGAGTCTGGAGTCTCAC TTGTCTCACTTGTG-3' and reverse, 5'-CATGGATCCGTC AGAGAATTTTGTTGCAAGCTTTATTTAG-3'. The PCR product was cloned using a TA cloning kit (Invitrogen Life Technologies), and the accuracy of the insert was established by Sanger sequencing. Subsequently, a QuickChange XL Site-Directed Mutagenesis kit (Agilent Technologies, Inc., Santa Clara, CA, USA) was used to introduce another allele of rs3783553 using the following primer sequence to delete TTCA: Forward, 5'-TTACCTGGGCATTCTTGTATTCCACCTGCA ATCAAG-3'; and complementary reverse, 5'-CTTGATTGC AGGTGGAATACAAGAATGCCCAGGTA A-3'. Finally, the 3'-UTR of Renilla luciferase in the pRL-SV40 vector (Promega Corporation, Madison, WI, USA) was substituted with the generated wild-type and mutant 3'-UTR of IL-1A. A luciferase assay was performed in the human synovial cells as previously described (26). Briefly, cells were seeded at $1 \times 10^{5}$ cells per well in 24-well plates. After $12 \mathrm{~h}$, the cells were transfected using Lipofectamine 2000 (Invitrogen Life Technologies) according to the manufacturer's instructions. Each well comprised the wild-type or mutant construct (500 ng) and pGL3 control vector (50 ng) that were co-transfected with 100 pmol pre-miR-122 or the 22-nt oligonucleotide negative control (Ambion Life Technologies, Austin, TX, USA). Following transfection (duration, $24 \mathrm{~h}$ ), the cells were harvested by the addition of a passive lysis buffer (Promega Corporation). Luciferase activity in the cell lysate was determined using the Dual-Luciferase Reporter assay system (Promega Corporation) in a TD-20/20 Luminometer (Turner Biosystems Inc., Sunnyvale, CA, USA).

Statistical analysis. Fisher's exact and $\chi^{2}$ tests, as well as Student's t-test were used to compare the frequency distribution of age, gender, body mass index (BMI) and smoking status between the OA cases and control subjects. The Hardy-Weinberg equilibrium (HWE) of the genotype frequencies of the controls was assessed using the $\chi^{2}$ test. The association between the rs 3783553 polymorphism and OA risk was estimated by computing odds ratios (ORs), and their 95\% confidence intervals (CIs) from unconditional logistic regression analysis with the adjustment for possible confounders. Student's t-test was used to evaluate the association between the rs3783553 genotype and SF concentration of miR122 and IL-1A. $\mathrm{P}<0.05$ was considered to indicate a statistically significant difference, and all analyses were performed using IBM SPSS 19.0 software (Armonk, NY, USA).

\section{Results}

rs3783553 genotypes affect IL-1A expression by regulating the miR-122 binding in human synovial cells. To investigate how the rs3783553 genotypes affect IL-1A expression, the 3'-UTR of a Renilla luciferase reporter gene was replaced with the full length IL-1A 3'-UTR containing either allele of the rs3783553 polymorphism. In transiently transfected human synovial cells, compared with the constructs containing TTCA Del alleles, luciferase activity from constructs containing the TTCA Ins allele was significantly lower in the presence of miR-122 (Fig. $1 \mathrm{~A}$ and $\mathrm{B} ; \mathrm{P}<0.01$ ). This result indicated that miR-122 binds to, and negatively regulates the transcription of, IL-1A and that this regulation is negatively influenced by the presence of the TTCA Ins allele, which is likely to affect the binding of miR-122 to the IL-1A transcript.

Exogenous expression of miR-122 suppresses the expression of IL-1A with Ins/Ins and Ins/Del genotypes, but not the Del/Del genotype. The synovial tissues of each rs3783553 genotype were obtained from the patients who received a synovectomy, and the cells were digested, passaged and cultured prior to the functional analysis. The endogenous expression levels of miR-122 and IL-1A were determined in each cell group using RT-qPCR and western blot analysis, and the expression of miR-122 and IL-1A mRNA was found to be comparable between each group (Fig. 1C and E); however, the IL-1A protein expression level in the Del/Del group was markedly higher than in the Ins/Ins and Ins/Del groups (Fig. 1D). Additionally, hsa-miR-122 mimics were transfected into the synovial cells of each genotype, and the mRNA and protein expression levels of IL-1A in the synovial cells were examined again. This demonstrated that the protein level of IL-1A in the Del/Del group was not changed; however in the Ins/Ins and Ins/Del groups, the protein level was substantially lowered (Fig. 2A) and the mRNA expression level remained unchanged (Fig. 2B).

Demographic characteristics. A total of 931 OA patients and 952 healthy control subjects were recruited; the demographic characteristics and $\mathrm{OA}$ radiographic severity are presented in 
A

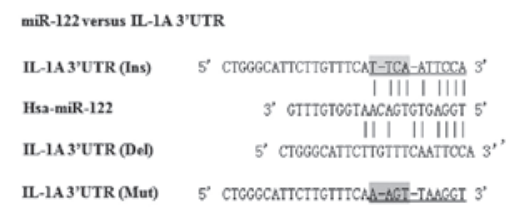

C

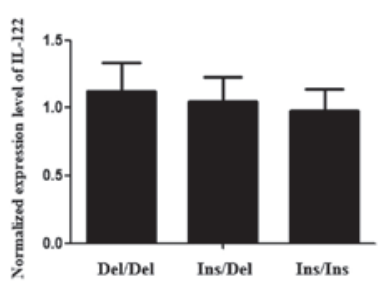

B

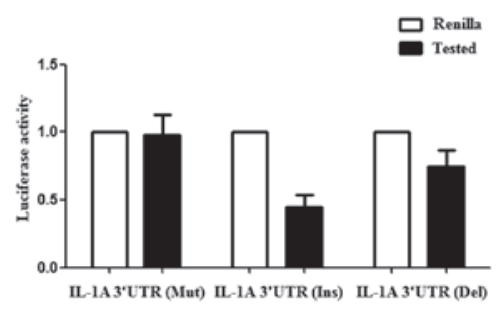

D

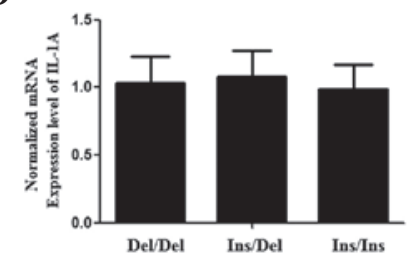

$\mathbf{E}$

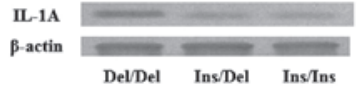

Figure 1. (A) Schematic representation of miR-122 and wild-type/Mut 3'-UTR of IL-1A. (B) Results of the luciferase assay comparing the inhibitory effect of miR-122 on the luciferase activity of synovial cells transfected with plasmids containing wild-type or Mut 3'-UTR of IL-1A. (C) Expression level of miR-122 in synovial cells obtained from the patients with different rs3783553 genotypes. (D) mRNA expression level of IL-1A in synovial cells obtained from the patients with different rs3783553 genotypes determined by reverse transcription-quantitative polymerase chain reaction. (E) Protein expression level of IL-1A in synovial cells obtained from the patients with different rs3783553 genotypes determined by western blot analysis. IL-1A, interleukin-1 $\alpha$; 3 '-UTR, 3'-untranslated region; Ins, insertion; Del, deletion; Mut, mutant.

A

$$
\text { IL-1A }
$$

B-actin

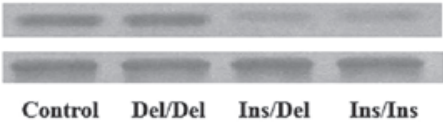

C

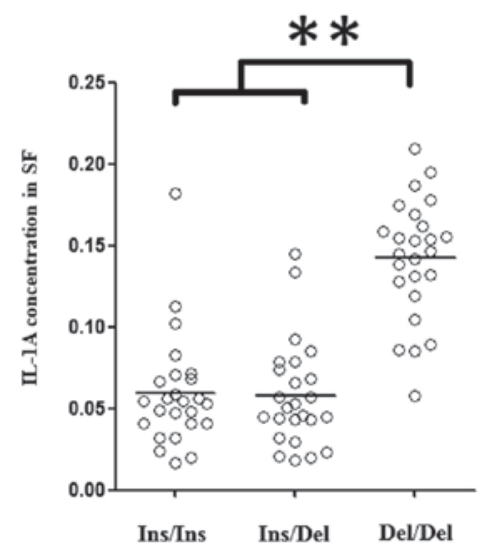

B

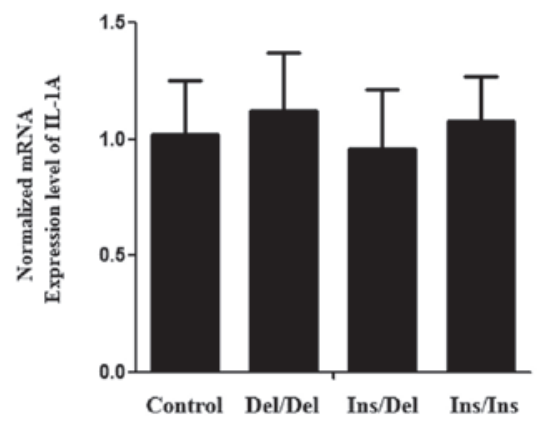

D

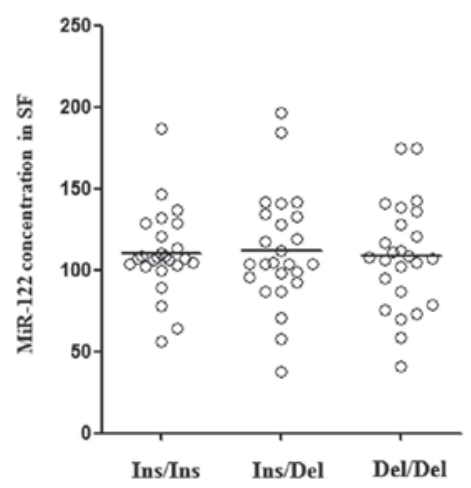

Figure 2. Analysis of synovial cells of each rs3783553 genotype that were transfected with miR-122 mimics to determine the (A) protein expression level of IL-1A by western blotting and (B) mRNA expression level of IL-1A by reverse transcription-quantitative polymerase chain reaction. (C) IL-1A and (D) miR-122 concentrations were measured in SF collected from subjects of each genotype. IL-1A, interleukin-1 $\alpha$; Del, deletion; Ins, insertion; SF, synovial fluid. 
Table I. Distribution of selected variables between the OA cases and control subjects.

\begin{tabular}{lll}
\hline Variable & OA, $\mathrm{n}=931$ & Control, $\mathrm{n}=952$ \\
\hline Gender, $\mathrm{n}(\%)$ & & \\
Male & $614(66)$ & $399(63)$ \\
Female & $317(34)$ & $353(37)$ \\
Age, years & $63.2 \pm 12.9$ & $62.6 \pm 11.2$ \\
Body mass index, $\mathrm{kg} / \mathrm{m}^{2}$ & $27.2 \pm 3.9$ & $24.6 \pm 3.7$ \\
Smoking status, $\mathrm{n}(\%)$ & & $609(64)$ \\
Yes & $633(68)$ & $343(36)$ \\
Never & $298(32)$ & \\
Kellgren-Lawrence score, $\mathrm{n}(\%)$ & & \\
Grade 1 and 2 & $670(72)$ & \\
Grade 3 and 4 & $261(28)$ & \\
\hline
\end{tabular}

OA, osteoarthritis.

Table II. Genotype and allele frequencies of the rs 3783553 polymorphism among cases and control subjects, and the associations with OA risk.

\begin{tabular}{lccr}
\hline rs3783553 polymorphism & Control, $\mathrm{n}=952(\%)$ & OA, $\mathrm{n}=931(\%)$ & OR (95\% CI) \\
\hline Del/Del & $114(12)$ & $158(17)$ & 1.00 \\
Ins/Del & $447(47)$ & $418(45)$ & $0.67(0.55-0.88)$ \\
Ins/Ins & $391(41)$ & $355(38)$ & $0.65(0.49-0.86)$ \\
Ins/Del + Ins/Ins & $838(88)$ & $773(83)$ & $0.66(0.51-0.86)$ \\
\hline
\end{tabular}

OA, osteoarthritis; OR, odds ratio; CI, confidence interval; Del, deletion; Ins, insertion.

Table I. No statistically significant differences were observed with respect to age $(\mathrm{P}=0.2599)$, gender $(\mathrm{P}=0.1698)$ and smoking status $(\mathrm{P}=0.0704)$. Higher $\mathrm{BMI}$ values were detected among the OA patients $(\mathrm{P}=0.0001)$.

Association of rs3783553 genotypes with the concentrations of $I L-1 A$ and miR-122 in SF. Three different genotypic synovial fluid samples were used to further investigate the impacts of rs3783553 on the transcription of IL-1A: 25 Homozygous for the TTCA Del allele, 25 heterozygous and 25 homozygous for the TTCA Ins allele. Using an ELISA detection kit, the IL-1A level observed in the TTCA Ins homozygous group was identified to be comparable to the heterozygous group, and the two were found to be notably lower than that of the TTCA Del homozygous group (Fig. 2C). To eliminate possible effects from miR-122, the concentration of miR-122 in SFs of the same 75 patients was measured, and no difference was identified between the IL-1A rs3783553 genotype groups (Fig. 2D).

Association of rs3783553 genotypes with the risk for OA and its radiographic severity. To analyze the association between the IL-1A rs3783553 polymorphism and the risk for OA in a Chinese Han population, $931 \mathrm{OA}$ patients and 952 healthy individuals were determined for the allelic and genotypic frequencies of the IL-1A rs3783553 polymorphism. The rs3783553 polymorphism was in HWE and was assessed for correlation with the risk for $\mathrm{OA}$ and its radiographic severity. A significant association was noted between the IL-1A rs3783553 polymorphism rare allele (Del) and an increased risk for OA [following adjustment for multiple potential risk factors (Table II)]. The frequency of the IL-1A rs3783553 polymorphism, Del allele was identified to be notably higher in the OA patients than in the control subjects (Table II), which was demonstrated by an allelic frequency analysis, following adjustment for known risk factors. Additionally, a significant association between rs3783553 homozygote (Del/Del) and an increased OA severity was noted in the recessive genetic model, compared with its heterozygote and homozygote (Table II; OR $=0.71,95 \% \mathrm{CI}=0.55-0.92$, and $\mathrm{P}=0.010$ ). Due to the relatively large sample size, a stratified analysis by patient age, gender, BMI and smoking status was performed. It was noted that the association was even more marked in young subjects $(<62$ years) (Table III; OR=0.53; $\mathrm{P}<0.001$ ).

\section{Discussion}

An important step toward the clinical application of this novel subclass of genetic variations in health management will be to improve understanding of distinct polymorphisms in miRNA-associated gene and protein expression regulation in a 
Table III. Stratification analysis between the different genotypes of the rs 3783553 polymorphism and the OA risk.

\begin{tabular}{|c|c|c|c|c|}
\hline \multirow[b]{2}{*}{ Variable } & \multicolumn{2}{|c|}{ Genotype, n } & \multirow[b]{2}{*}{$\begin{array}{c}\text { Ins/Del + Ins/Ins vs. Del/Del } \\
{[\text { OR }(95 \% \text { CI })]}\end{array}$} & \multirow[b]{2}{*}{ P-value } \\
\hline & $\begin{array}{c}\text { Del/Del } \\
\text { (Case/Control) }\end{array}$ & $\begin{array}{l}\text { Ins/Del + Ins/Ins } \\
\text { (Case/Control) }\end{array}$ & & \\
\hline \multicolumn{5}{|c|}{ Age, years } \\
\hline$\leq 62$ & $78 / 54$ & $366 / 460$ & $0.53(0.36-0.77)$ & 0.0010 \\
\hline$>62$ & $80 / 60$ & $407 / 378$ & $0.78(0.54-1.13)$ & 0.018 \\
\hline \multicolumn{5}{|l|}{ Gender } \\
\hline Male & $104 / 72$ & $510 / 528$ & $0.66(0.48-0.92)$ & 0.015 \\
\hline Female & $54 / 42$ & $263 / 310$ & $0.66(0.42-1.02)$ & 0.061 \\
\hline \multicolumn{5}{|l|}{ Smoker } \\
\hline Never & $107 / 72$ & $510 / 528$ & $0.69(0.50-0.96)$ & 0.028 \\
\hline Yes & $51 / 42$ & $263 / 310$ & $0.70(0.45-1.09)$ & 0.110 \\
\hline \multicolumn{5}{|l|}{ BMI } \\
\hline$\leq 25$ & $55 / 59$ & $271 / 435$ & $0.66(0.44-1.00)$ & 0.047 \\
\hline$>25$ & $103 / 55$ & $502 / 403$ & $0.67(0.47-0.95)$ & 0.023 \\
\hline Total & $158 / 114$ & $773 / 838$ & $0.66(0.51-0.86)$ & 0.0021 \\
\hline
\end{tabular}

Del, deletion ; Ins, insertion; OR, odds ratio; CI, confidence interval; BMI, body mass index.

variety of diseases at the population level. In the present study, performance of a luciferase assay demonstrated that rs3783553 genotypes affect IL-1A expression by regulating the miR-122 binding in human synovial cells. Furthermore, the rs3783553 polymorphism was shown to be significantly associated with $\mathrm{OA}$ risk in a Chinese Han population $(\mathrm{OR}=0.71 ; \mathrm{P}=0.010)$, and an even more marked association was noted in younger subjects $(\mathrm{OR}=0.45 ; \mathrm{P}<0.001)$. Additionally, it was found that the genotype was associated with the radiographic severity in the same population $(\mathrm{OR}=0.72 ; \mathrm{P}=0.023)$.

Modulation of the immune system depends on the complex interaction within the cytokine network, where an optimal physiological environment must be maintained by the delicate modulation of the products of cytokine genes and their respective receptor genes. The IL-1 system is an effective example of the highly regulated mechanism, which is necessary for this to be achieved. An important signaling pathway in the inflammatory process, innate immunity and the immune response is IL-1 signaling via the IL-1 receptor type I (IL-1R) (32). It has various effects, including the stimulation of fibroblasts, keratinocyte proliferation, cartilage breakdown, angiogenesis and incremental production of acute phase response proteins by the liver (32). The activation of c-Jun N-terminal kinases and p38 mitogen-activated protein kinases and subsequent upregulation of the expression of genes via the transcription factors, nuclear factor- $\kappa$-light-chain-enhancer of activated B cells, activator protein-1 and CCAAT-enhancer-binding proteins, mediate these physiological effects at the molecular level (33). It is hypothesized that the products of the IL-1 gene cluster are involved in various diseases, one of which is OA. IL-1 may stimulate chondrocytes to produce cartilage matrix degrading enzymes, thereby contributing to this loss. In support of this hypothesis, the specific cleavage of the aggrecan core protein at the Glu373-Ala374 bond was found to be strongly correlated with the release of aggrecan catabolites in response to IL-1 (34). The SFs of OA patients are known to possess fragments bearing this epitope (35). Furthermore, compared with normal synovium, cells from OA synovium secrete more IL-1b $(36,37)$, and similarly, chondrocytes from OA cartilage produce IL-1A and IL-1b that is quantitatively different from normal chondrocytes $(38,39)$. Compared with similar explants from non-arthritic cartilage (40), OA cartilage explants tend to be more susceptible to the effects of IL-1, and the expression of IL-1R and $-2 \mathrm{R}$ on chondrocytes is relevant to this susceptibility (41).

The risk allele is likely to be more prevalent during early tumor development, which creates an improved binding site for miR-122, and inhibits IL-1A expression and immune surveillance. Gao et al performed an in-silicon analysis, and predicted that miR-122 and miR-378 were binding tightly to IL-1A mRNA transcripts, which contain the TTCA Del allele and modulate IL-1A expression in a negative manner (26). By contrast, binding with mRNA transcripts, which contain the TTCA Ins allele would be interrupted, causing upregulation of IL-1A expression. The luciferase assay, which compared IL-1A 3'-UTR with different rs3783553 genotypes was in support of this hypothesis, revealing the following relative transcription activities: Del/Del, 1.0; Del/Ins, 3.8; and Ins/Ins, 5.5 (17). Consistently, the highest IL-1A mRNA level was detected in the TTCA Ins homozygous group, followed by the heterozygous and TTCA Del groups, as demonstrated by a TaqMan ${ }^{\circledR}$ Gene Expression analysis of the tumor tissues of different rs3783553 genotypes from hepatocellular carcinoma patients. When compared with the TTCA Del homozygous group, the average IL-1A concentrations were 3.76- and 5.57-fold higher in the heterozygous and TTCA Ins homozygous 
groups, respectively, and the average IL-1A expression levels were 3.76- and 5.57-fold higher in the heterozygous and TTCA Ins homozygous groups, respectively. In the present study, a luciferase reporter system was used to establish IL-1A as an effective target gene of miR-122 in synovial cells that were obtained from patients who had received a synovectomy. This finding was further validated by the observation that exogenous over-expression of miR-122 in the synovial cells significantly downregulated the expression of IL-1A in the cells with Ins/Ins and Ins/Del genotypes, although this was not observed in the Del/Del group. Furthermore, the expression pattern of miR-122 and IL-1A in the synovial cells and SF of different genotypes was examined, and the miR-122 concentration was identified to be comparable among each group, although the IL-1A expression levels in the Ins/Ins and Ins/Del genotype groups were markedly lower than those of the Del/Del group in the synovial cells and the SF.

In conclusion, the risk caused by a polymorphism in the IL-1A gene and a potential biological mechanism for an increased risk of OA have been identified in the present study via genetic association. Due to the high expression of miR-122 in human synovium, further studies are required to determine whether miR-122 and IL-1A may be applied to the diagnosis and treatment of $\mathrm{OA}$.

\section{References}

1. Dinarello CA: The interleukin-1 family: 10 years of discovery. FASEB J 8: 1314-1325, 1994.

2. Lotz M, Blanco FJ, von Kempis J, Dudler J, Maier R, Villiger PM and Geng Y: Cytokine regulation of chondrocyte functions. J Rheumatol Suppl 43: 104-108, 1995.

3. van den Berg WB, Joosten LA and van de Loo FA: TNF alpha and IL-1 beta are separate targets in chronic arthritis. Clin Exp Rheumatol 17 (6 Suppl 18): S105-S114, 1999.

4. Luo L, Cruz T and McCulloch C: Interleukin 1-induced calcium signalling in chondrocytes requires focal adhesions. Biochem J 324: 653-658, 1997.

5. Pritchard S and Guilak F: Effects of interleukin-1 on calcium signaling and the increase of filamentous actin in isolated and in situ articular chondrocytes. Arthritis Rheum 54: 2164-2174, 2006.

6. Hubbard JR, Steinberg JJ, Bednar MS and Sledge CB: Effect of purified human interleukin-1 on cartilage degradation. J Orthop Res 6: 180-187, 1988.

7. Kahle P, Saal JG, Schaudt K, Zacher J, Fritz P and Pawelec G: Determination of cytokines in synovial fluids: correlation with diagnosis and histomorphological characteristics of synovial tissue. Ann Rheum Dis 51: 731-734, 1992.

8. Apte RN and Voronov E: Is interleukin-1 a good or bad 'guy' in tumor immunobiology and immunotherapy? Immunol Rev 222 222-241, 2008.

9. McNulty AL, Rothfusz NE, Leddy HA and Guilak F: Synovial fluid concentrations and relative potency of interleukin-1 alpha and beta in cartilage and meniscus degradation. J Orthop Res 31: 1039-1045, 2013.

10. Henderson B and Pettipher ER: Arthritogenic actions of recombinant IL-1 and tumor necrosis factor alpha in the rabbit: evidence for synergistic interactions between cytokines in vivo. Clin Exp Immunol 75: 306-310, 1989.

11. Smith AJ, Keen LJ, Billingham MJ, Perry MJ, Elson CJ, Kirwan JR, Sims JE, Doherty M, Spector TD and Bidwell JL: Extended haplotypes and linkage disequilibrium in the IL1R1-IL1A-IL1B-IL1RN gene cluster: association with knee osteoarthritis. Genes Immun 5: 451-460, 2004.

12. Valdes AM and Spector TD: The contribution of genes to osteoarthritis. Rheum Dis Clin North Am 34: 581-603, 2008.

13. Valdes AM and Spector TD: Genetic epidemiology of hip and knee osteoarthritis. Nat Rev Rheumatol 7: 23-32, 2011.

14. Pillai RS: MicroRNA function: multiple mechanisms for a tiny RNA? RNA 11: 1753-1761, 2005.
15. Wiemer EA: The role of microRNAs in cancer: no small matter. Eur J Cancer 43: 1529-1544, 2007.

16. Chen K, Song F, Calin GA, Wei Q, Hao X and Zhang W: Polymorphisms in microRNA targets: a gold mine for molecular epidemiology. Carcinogenesis 29: 1306-1311, 2008.

17. Yu Z, Li Z, Jolicoeur N, Zhang L, Fortin Y, Wang E, Wu M and Shen SH: Aberrant allele frequencies of the SNPs located in microRNA target sites are potentially associated with human cancers. Nucleic Acids Res 35: 4535-4541, 2007.

18. Saunders MA, Liang H and Li WH: Human polymorphism at microRNAs and microRNA target sites. Proc Natl Acad Sci USA 104: 3300-3305, 2007.

19. Chin LJ, Ratner E, Leng S, Zhai R, Nallur S, Babar I, Muller RU, Straka E, Su L, Burki EA, et al: A SNP in a let-7 microRNA complementary site in the KRAS 3 'untranslated region increases non-small cell lung cancer risk. Cancer Res 68: 8535-8540, 2008.

20. Landi D, Gemignani F, Naccarati A, Pardini B, Vodicka P, Vodickova L, Novotny J, Försti A, Hemminki K, Canzian F and Landi S: Polymorphisms within micro-RNA-binding sites and risk of sporadic colorectal cancer. Carcinogenesis 29: 579-584, 2008.

21. Brendle A, Lei H, Brandt A, Johansson R, Enquist K, Henriksson R, Hemminki $K$, Lenner $P$ and Försti $A$ : Polymorphisms in predicted microRNA-binding sites in integrin genes and breast cancer: ITGB4 as prognostic marker. Carcinogenesis 29: 1394-1399, 2008.

22. Wang G, van der Walt JM, Mayhew G, Li YJ,Züchner S, Scott WK, Martin ER and Vance JM: Variation in the miRNA-433 binding site of FGF20 confers risk for Parkinson disease by overexpression of alpha-synuclein. Am J Hum Genet 82: 283-289, 2008.

23. Sethupathy P, Borel C, Gagnebin M, Grant GR, Deutsch S, Elton TS, Hatzigeorgiou AG and Antonarakis SE: Human microRNA-155 on chromosome 21 differentially interacts with its polymorphic target in the AGTR1 3' untranslated region: a mechanism for functional single-nucleotide polymorphisms related to phenotypes. Am J Hum Genet 81: 405-413, 2007.

24. Clop A, Marcq F, Takeda H, Pirottin D, Tordoir X, Bibé B, Bouix J, Caiment F, Elsen JM, Eychenne F, et al: A mutation creating a potential illegitimate microRNA target site in the myostatin gene affects muscularity in sheep. Nat Genet 38: 813-818, 2006.

25. Martin MM, Buckenberger JA, Jiang J, Malana GE, Nuovo GJ, Chotani M, Feldman DS, Schmittgen TD and Elton TS: The human angiotensin II type 1 receptor $+1166 \mathrm{~A} / \mathrm{C}$ polymorphism attenuates microRNA-155 binding. J Biol Chem 282: 24262-24269, 2007.

26. Gao Y, He Y, Ding J, Wu K, Hu B, Liu Y, Wu Y, Guo B, Shen Y, Landi D, et al: An insertion/deletion polymorphism at miRNA-122-binding site in the interleukin-1alpha 3' untranslated region confers risk for hepatocellular carcinoma. Carcinogenesis 30: 2064-2069, 2009.

27. Fermor B, Jeffcoat D, Hennerbichler A, Pisetsky DS, Weinberg JB and Guilak F: The effects of cyclic mechanical strain and tumor necrosis factor alpha on the response of cells of the meniscus. Osteoarthritis Cartilage 12: 956-962, 2004.

28. Kuettner KE, Pauli BU, Gall G, Memoli VA and Schenk RK: Synthesis of cartilage matrix by mammalian chondrocytes in vitro .1. Isolation, culture characteristics and morphology. J Cell Biol 93: 743-750, 1982.

29. Maroudas A and Evans H: A study of ionic equilibria in cartilage. Connect Tissue Res 1: 69-77, 1972.

30. Dayer JM, Krane SM, Russell RG and Robinson DR: Production of collagenase and prostaglandins by isolated adherent rheumatoid synovial cells. Proc Natl Acad Sci USA 73: 945-949, 1976

31. Attur M, Wang HY, Kraus VB, Bukowski JF, Aziz N, Krasnokutsky S, Samuels J, Greenberg J, McDaniel G, Abramson SB and Kornman KS: Radiographic severity of knee osteoarthritis is conditional on interleukin 1 receptor antagonist gene variations. Ann Rheum Dis 69: 856-861, 2010.

32. Dinarello CA: Biologic basis for interleukin-1 in disease. Blood 87: 2095-2147, 1996.

33. O'Neill LA and Greene C: Signal transduction pathways activated by the IL-1 receptor family: ancient signaling machinery in mammals, insects, and plants. J Leukoc Biol 63: 650-657, 1998.

34. Arner EC, Hughes CE, Decicco CP, Caterson B and Tortorella MD: Cytokine-induced cartilage proteoglycan degradation is mediated by aggrecanase. Osteoarthritis Cartilage 6: 214-228, 1998.

35. Fosang AJ, Last K, Knäuper V, Murphy G and Neame PJ: Degradation of cartilage aggrecan by collagenase-3 (MMP-13). FEBS Lett 380: 17-20, 1996. 
36. Westacott CI, Taylor G, Atkins R and Elson C: Interleukin 1 alpha and beta production by cells isolated from membranes around aseptically loose total joint replacements. Ann Rheum Dis 51: 638-642, 1992.

37. Elson CJ, Mortuza FY, Perry MJ, Warnock MG, Webb GR and Westacott CI: Cytokines and focal loss of cartilage in osteoarthritis. Br J Rheumatol 37: 106-107, 1998.

38. Towle CA, Hung HH, Bonassar LJ, Treadwell BV and Mangham DC: Detection of interleukin-1 in the cartilage of patients with osteoarthritis: a possible autocrine/paracrine role in pathogenesis. Osteoarthritis Cartilage 5: 293-300, 1997.
39. Moos V, Rudwaleit M, Herzog V, Höhlig K, Sieper J and Müller B: Association of genotypes affecting the expression of interleukin-1beta or interleukin-1 receptor antagonist with osteoarthritis. Arthritis Rheum 43: 2417-2422, 2000.

40. Ismaiel S, Atkins RM, Pearse MF, Dieppe PA and Elson CJ: Susceptibility of normal and arthritic human articular cartilage to degradative stimuli. Br J Rheumatol 31: 369-373, 1992.

41. Martel-Pelletier J, McCollum R, DiBattista J, Faure MP, Chin JA, Fournier S, Sarfati M and Pelletier JP: The interleukin-1 receptor in normal and osteoarthritic human articular chondrocytes. Identification as the type I receptor and analysis of binding kinetics and biologic function. Arthritis Rheum 35: 530-540, 1992. 